\title{
Nuevos aportes a la historia natural de la mulita pampeana Dasypus hybridus (Mammalia, Dasypodidae)
}

\author{
Agustín M. Abba ${ }^{1}$, Guillermo H. Cassini ${ }^{2,3}$ \& Fernando C. Galliari ${ }^{3}$
}

1. División Zoología Vertebrados, Facultad de Ciencias Naturales y Museo, Universidad Nacional de La Plata, Paseo del Bosque s/n, 1900 La Plata, Argentina. CONICET. abbaam@yahoo.com.ar
2. Departamento de Ciencias Básicas, Universidad Nacional de Luján, Rutas 5 y 7, 6700 Luján, Argentina. gcassini@unlu.edu.ar
3. División Paleontología Vertebrados, Facultad de Ciencias Naturales y Museo, Universidad Nacional de La Plata, Paseo del Bosque s/n, 1900 La Plata, Argentina.CONICET. fgalliari@hotmail.com

ABSTRACT. New contributions to the natural history of the southern long-nosed armadillo Dasypus hybridus (Mammalia, Dasypodidae). This contribution presents new data about the natural history of the Dasypus hybridus (Desmarest, 1804) (Mammalia, Xenarthra, Dasypodidae). A seasonal monitoring using the capture and release method was performed over the course of three years in a 100 ha of four cattle farms in the Buenos Aires province, Argentina. Data were collected on food habits, space and time use, behavior, thermoregulation, population data, and morphology. A total of 71 captures were made. The main food items recorded were plant matter, ants and coleopterans, and did not occur seasonal differences in food habits. Dasypus hybridus concentred their activities during daytime, and were recorded a low frequency of activities during cold seasons (fall and winter). The long-nosed armadillo prefer humid soils and grasslands with high vegetation cover, and selected forest to refuge. The southern long-nosed armadillo were asocial. Rectal temperature was positively correlated with environmental temperature. The sex proportion was near to one, and no sexual dimorphism was observed. In general, the results of this study are in agreement with previous observations of other species of the same genera, but is necessary to highlight the trend observed in food habits and thermoregulatory strategy. This work provides new information about different aspects of a poor studied species that occur in a habitat subjected to high pressure to modification and use.

KEYWORDS. Armadillos, Argentina, Cingulata, conservation, ecology.

RESUMEN. En este trabajo se presentan nuevos aportes sobre la historia natural de la mulita pampeana Dasypus hybridus (Desmarest, 1804) (Mammalia, Xenarthra, Dasypodidae). Los estudios de campo fueron llevados a cabo en 100 ha de cuatro establecimientos agropecuarios de la provincia de Buenos Aires, Argentina. Durante tres años se realizó un muestreo estacional de armadillos por captura y liberación. Se obtuvieron datos de hábitos alimentarios, uso del espacio y del tiempo, comportamiento, termorregulación, datos poblacionales y morfológicos. Se realizaron 71 capturas. En la dieta el ítem principal registrado fue material vegetal, seguido por hormigas e insectos coleópteros; no se observó una diferencia estacional en los hábitos alimentarios. La actividad de las mulitas se concentra durante el día, existió una baja en la frecuencia de observación durante las estaciones frías (otoño e invierno). La mulita pampeana prefiere suelos húmicos, terrenos altos y pastizales densos y altos; asimismo seleccionan los montes para refugiarse. Son individuos asociales. La temperatura rectal mostró correlaciones positivas con la temperatura ambiente. La proporción de sexos fue cercana a uno y no se observó dimorfismo sexual. Los resultados obtenidos concuerdan parcialmente con lo observado para otras especies del género, destacando las tendencias observadas en los hábitos alimentarios y en la estrategia termorregulatoria. Este trabajo representa un aporte en varios aspectos de una especie poco estudiada en una zona bajo importantes presiones de uso y modificación de hábitat.

PALABRAS-CLAVE. Armadillos, Argentina, Cingulata, conservación, ecología.

Dasypus, con sus siete especies, representa el género más numeroso y ampliamente distribuido de los xenartros actuales (Mammalia: Xenarthra, Wetzel et al., 2007). En América del Sur residen todas las especies del género, las cuales se distribuyen en una amplia variedad de ambientes, presentan hábitos semifosoriales y poseen una estrecha relación con las áreas donde se realizan actividades agropecuarias (REDFORD \& EISENBERG, 1992). Sin embargo, excepto por los estudios realizados en Dasypus novemcinctus Linnaeus, 1758 (McDonough \& LOUGHRY, 2008), se ha generado muy poca información metódica sobre aspectos generales de la biología de otras especies del género (ABBA \& CASSINI, 2008; McDonough $\&$ Loughry, 2008). En el presente trabajo se aporta información sobre la historia natural de una de las especies de mulita menos estudiada, la mulita pampeana u orejuda, Dasypus hybridus (Desmarest, 1804).

La distribución de la mulita pampeana abarca el sur de Paraguay, todo Uruguay, sur de Brasil y este de Argentina, encontrándose fundamentalmente en áreas abiertas cubiertas de pastizales (WETZEL, 1985; REDFORD \& EisenBerg, 1992; GonzÁlez et al., 2001; AbBA et al., 2007). Según modelos de ocupación de nicho su rango de distribución es restringido y sólo se la encuentra en las regiones de la Pampa y de la Mata Atlántica
(ANACLETo et al., 2006). Asimismo, se la ha registrado en agroecosistemas, tanto de origen ganadero como agrícola (ABBA et al., 2007).

Existen pocos trabajos de historia natural y ecología cuyo objeto de estudio haya sido esta especie (Abba \& Cassini, 2008; McDonough \& Loughry, 2008). GonZÁlez et al. (2001) realizaron un estudio en Uruguay que analiza la distribución, la estructura y el estado de uso de las cuevas; CASANAVE et al. (2003) realizaron un estudio de la comunidad de armadillos en el sur de la provincia de Buenos Aires e hicieron aportes sobre los períodos de actividad de la mulita. En cuanto a la composición de la dieta en esta especie, sólo se han hecho aportes preliminares (ver ABBA \& CASSINI, 2008). AввA et al. (2007), a partir de datos de evidencias indirectas registradas en el noreste de la provincia de Buenos Aires, aportaron varios aspectos sobre uso y conservación. ABBA \& CASSINI (2008), en un artículo de revisión, presentan varias cuestiones por resolver de la especie. Finalmente, ABBA \& CASSINI (2010) analizaron distintas dimensiones del nicho y explicaron la segregación que ocurre con Chaetophractus vellerosus Gray, 1865 en áreas de simpatría.

Desde el punto de vista de los mecanismos reproductivos, este armadillo presenta varios rasgos 
peculiares: posee poliembronía específica obligada, siendo sus crías hermanos idénticos o clones, es decir, derivados todos de un solo huevo fertilizado. Asociada a esta particularidad, y siendo una de las pocas excepciones dentro de los mamíferos, tiene numerosas crías aunque sólo posee cuatro mamas. Además, tiene la capacidad de una implantación diferida de los embriones (FERNANDEZ, 1915; CABrera \& YePEs, 1940; Wetzel, 1985).

Este trabajo tiene como objetivo general realizar nuevos aportes sobre la historia natural de la mulita pampeana, D. hybridus. Los objetivos específicos del mismo son aportar información referente a hábitos alimentarios, uso del espacio y del tiempo, comportamiento, termorregulación, reproducción y morfometría.

\section{MATERIALES Y MÉTODOS}

Área de estudio y muestreo. Este estudio se realizó durante tres años (desde febrero 2006 a febrero 2009) en cuatro establecimientos agropecuarios ubicados en tres localidades de la provincia de Buenos Aires, Argentina

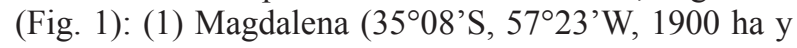
$35^{\circ} 10^{\prime} \mathrm{S}, 57^{\circ} 20^{\prime} \mathrm{W}, 650$ ha, ambos establecimientos son tomados como una sola población de mulita pampeana ya que se encuentran muy cercanas entre sí, a unos $7 \mathrm{~km}$ aproximadamente); (2) Punta Indio (35 $33^{\prime} \mathrm{S}, 57^{\circ} 14^{\prime} \mathrm{W}$, 1000 ha) y (3) Pellegrini (36 $17^{\prime}$ S, 632' $\mathrm{W}, 1900$ ha).

La zona donde se realizó el estudio, pastizales pampeanos, es la región más transformada de Argentina y una de las más modificadas del mundo (ver BiLENCA \& MiÑARO, 2004). En términos generales, los pastizales pampeanos pueden ser considerados como una vasta

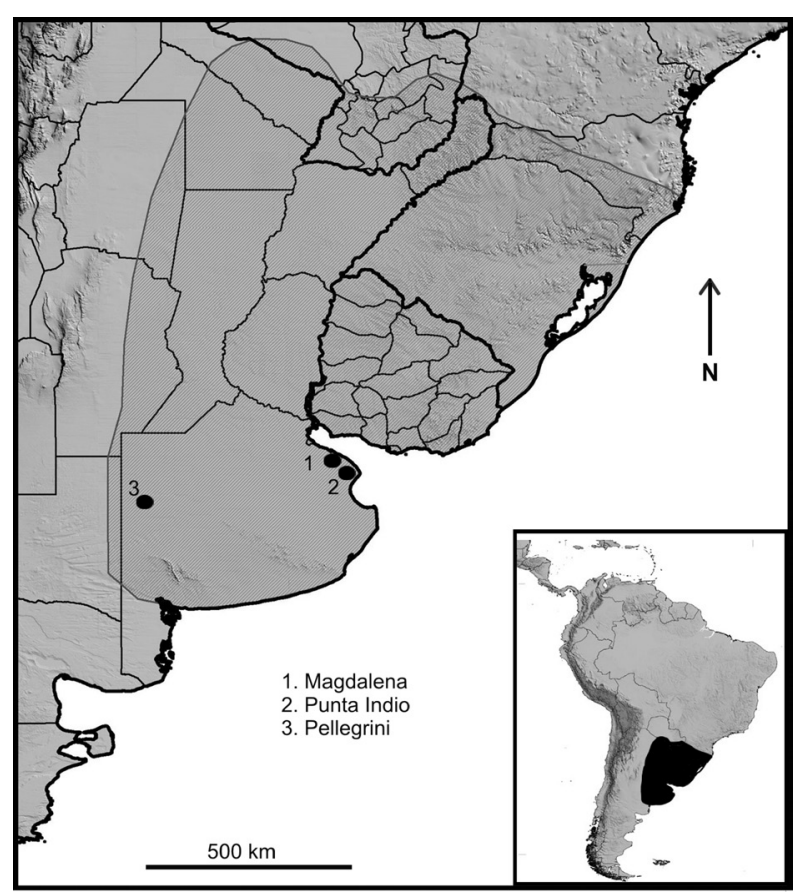

Fig. 1. Mapa con las localidades en la Provincia de Buenos Aires, Argentina donde se hicieron capturas de Dasypus hybridus (Desmarest, 1804) durante febrero 2006 a febrero 2009. En sombreado se presenta la distribución de la especie (modificado de ABBA \& SuPERINA, 2010). y continua planicie en la que se alternan, a lo largo de grandes distancias, paisajes totalmente planos con otros de relieve ligeramente ondulado. La región posee un clima templado de tipo subhúmedo a seco en el oeste y de tipo húmedo en el este. Las precipitaciones varían entre los $1000 \mathrm{~mm}$ anuales en el NE hasta los $500 \mathrm{~mm}$ anuales en el SO. Desde el punto de vista térmico, la región posee características mesotérmicas, con una temperatura media anual que oscila entre los $14^{\circ} \mathrm{C}$ en el sur y los $18^{\circ} \mathrm{C}$ en el norte (Bilenca \& Miñaro, 2004). Los suelos pampeanos por lo general están compuestos de materiales finos - arenas, loes, limos y arcillas encontrando principalmente suelos arenosos, calcáreoarenosos, arcillosos y húmicos. La región pampeana se caracteriza por la preponderancia de pastizales o estepas graminosas cuya comunidad clímax es la pradera de flechilla o flechillar, extendiéndose sobre campos altos con suelos húmicos y arcilloso-arenoso ligeramente ácidos (Bilenca \& MiñARo, 2004). En la zona de Magdalena y Punta Indio encontramos una comunidad subclimáxica denominada Bosque Xerófilo Periestépico. Esta formación, condicionada por el factor suelo, se extiende en delgadas fajas paralelas a la rivera platense, aprovechando los cordones o albardones de conchillas. Fisonómicamente, se trata de un bosque bajo, formado por árboles achaparrados, de tres a seis metros de altura, con sotobosque de arbustos y hierbas. Las especies dominantes (Celtis tala y Acacia caven) son algo xeromorfas, con hojas coriáceas, reducidas e incluso áfilas. Los establecimientos donde fueron realizados los muestreos son dedicados a la ganadería y en menor medida a la agricultura, estas actividades agregan a los pastizales y montes naturales áreas con pasturas para el ganado y rastrojos principalmente de trigo y de soja.

Los trabajos de campo se basaron en muestreos que consistían en recorrer durante todo el día (de 12:00 a 24:00 hs) 100 ha de los campos seleccionados capturando y liberando todas las mulitas que se observaban. El esfuerzo de muestreo fue similar entre las distintas áreas y los sucesivos años, excepto en Pellegrini donde no se realizaron campañas en invierno ni verano (ver Tabla I), resultando en un total de 80 días de campaña, divididos en 20 días por estación del año. La técnica de captura utilizada fue manual (handling) y con la ayuda de una red; se seleccionó esta metodología debido a que los armadillos no suelen caer en las trampas estándar, además permite obtener la información necesaria de manera rápida y sin dañar a los individuos. Como complemento a esta búsqueda, se exploraban minuciosamente las cuevas encontradas para poder capturar los individuos que estuvieran dentro.

Todos los análisis estadísticos se realizaron con el programa de estadística R (R Development Core Team, 2010).

Hábitos alimentarios. Se colectaron 32 fecas de individuos capturados en los partidos de Magdalena y Punta Indio (tres en invierno, cinco en otoño, 15 en primavera y nueve en verano). Para el análisis se siguieron las técnicas propuestas a partir de bibliografía 
Tab. I. Localidades, épocas del año y cantidad de mulitas pampeanas (Dasypus hybridus) capturadas en la Provincia de Buenos Aires, Argentina, según edad y sexo (A, adulto; J, juvenil; C, cría).

\begin{tabular}{|c|c|c|c|c|c|}
\hline & Otoño & Invierno & Primavera & Verano & TOTAL \\
\hline Magdalena & $1 \mathrm{~A}+$ & $\begin{array}{c}1 \mathrm{~J} q \\
1 \uparrow \mathrm{A}\end{array}$ & $\begin{array}{c}4 \mathrm{~A} \\
(3 \lesssim 1+)\end{array}$ & $\begin{array}{c}4 \mathrm{~J} \delta \\
7 \mathrm{~A}\left(5{ }^{\lambda} 29\right)\end{array}$ & $5 \mathrm{~J}+13 \mathrm{~A}=18$ \\
\hline Punta Indio & $\begin{array}{c}4 \mathrm{~A} \\
\left(2 \Im^{\lambda} 2+\right)\end{array}$ & $\begin{array}{c}1 \mathrm{~J} \delta \\
5 \mathrm{~A}\left(2{ }^{\lambda} 3+\right)\end{array}$ & $\begin{array}{c}14 \mathrm{C}^{\circ} \\
10 \mathrm{~A}\left(5 \delta^{\lambda} 5 q\right)\end{array}$ & $\begin{array}{c}2 \mathrm{~J}+ \\
7 \mathrm{~A}\left(3{ }^{\lambda} 49\right)\end{array}$ & $14 \mathrm{C}+3 \mathrm{~J}+26 \mathrm{~A}=43$ \\
\hline Pellegrini & $\begin{array}{l}2 \mathrm{~J}_{\bigcirc}^{\lambda} \\
2 \mathrm{~A} \text { ㅇ }\end{array}$ & - & $\begin{array}{c}2 \mathrm{~J}\left(1{ }^{\Uparrow} 1+\right) \\
4 \mathrm{~A}+\end{array}$ & - & $4 \mathrm{~J}+6 \mathrm{~A}=10$ \\
\hline TOTAL & $2 \mathrm{~J}+7 \mathrm{~A}$ & $2 J+6 \mathrm{~A}$ & $14 \mathrm{C}+2 \mathrm{~J}+18 \mathrm{~A}$ & $6 \mathrm{~J}+14 \mathrm{~A}$ & $14 \mathrm{C}+12 \mathrm{~J}+45 \mathrm{~A}=71$ \\
\hline
\end{tabular}

existente (Greegor, 1980; Anacleto \& Marinho-Filho, 2001; ANACleto, 2007). Las muestras fueron estudiadas bajo lupa binocular de entre 1 y 4 aumentos. Para la determinación sistemática del material encontrado en las heces se realizó una colección de referencia y se utilizó bibliografía específica de cada grupo (Gerschman de Pikelín \& Schiapelli, 1963; Orrego Aravena, 1974; Morrone \& Coscarón, 1998; Cicchino, 2006). Los resultados se expresan en porcentaje en volumen $(\% \mathrm{~V}$, ver OTtino \& GiLleR, 2004 y referencias allí citadas). Los ítems registrados en este análisis se clasificaron en distintas categorías de alimento (ver Resultados). Se estimó la frecuencia de ocurrencia de cada categoría discriminada por estación del año. La dependencia de dichas frecuencias se evaluó mediante tablas de contingencia multidimensionales siguiendo los procedimientos descritos en ZAR (1999).

Uso del espacio y del tiempo. Para determinar el modo de uso del espacio y del tiempo por la mulita pampeana, se tuvieron en cuenta 45 capturas (ver Tabla I individuos adultos) realizadas en los cuatro campos. Sólo se contabilizaron las capturas de individuos adultos que estaban activos, descartándose las mulitas capturadas dentro de las cuevas. Para estos análisis se utilizaron las siguientes variables: fecha, hora, tipo de suelo (húmicos y arenoso-arcillosos, siguiendo las descripciones provistas por el Soll Survey Staff, 1999), ubicación con respecto a las variaciones micro topográficas del terreno (tope o bajo), vegetación del lugar donde se realizó la captura (Tipo: pastizal - pastura - monte - rastrojo, Cobertura: alta $>50 \%$ de suelo cubierto baja $<50 \%$ y Altura: alta $>20 \mathrm{~cm}-$ baja $<$ a $20 \mathrm{~cm}$ ) y georreferenciación (GPS). La diferencia en la cantidad de capturas por estación, como así también por tipo de suelo se evaluaron mediante pruebas $\chi^{2}$ (Chi-cuadrado) para proporciones. El análisis estacional de los horarios de captura se realizó mediante estadística circular. Los datos en horas se convirtieron en ángulos para ser evaluados mediante una distribución circular (MENA, 2004). El ajuste de los datos a una distribución angular uniforme se evaluó mediante la prueba $\mathrm{Z}$ de Raileight y las diferencias entre las medias de horario de captura por estación se evaluaron mediante la prueba de WatsonWilliams para comparaciones múltiples (ZAR, 1999).

Comportamiento. Se tuvieron en cuenta los registros de "comportamiento inicial", es decir, de la actividad del animal al momento en que el observador lo detectaba, también se anotaba si se encontraba sólo o en grupo. Las pautas definidas para el comportamiento fueron: quieto (cuando un animal estaba parado sin realizar movimiento alguno), andando (cuando estaba desplazándose, tanto corriendo como caminado), hozando (cuando estaban alimentándose o en búsqueda del alimento, cavando, moviendo la tierra con el hocico, oliendo el suelo), y en cueva (cuando un animal estaba dentro de su refugio). Se utilizaron 55 registros de pautas comportamentales previas a la captura. Asimismo se registró el comportamiento de liberación o huída. Se liberaba el animal en el mismo lugar de captura y se lo seguía a una distancia prudencial $(15-20 \mathrm{~m}$ como mínimo), para no afectar el normal comportamiento de los individuos, de forma tal que fuera posible determinar el tipo de ambiente en el cual el individuo se refugiaba. Para este análisis de huída se evaluó la disponibilidad de los ambientes; se utilizaron imágenes satelitales de los campos para diferenciar entre monte y pastizal natural/pastura; una vez obtenida la imagen se realizó una cuadrícula y se estimó la proporción de superficie de cada ambiente. Se aplicaron pruebas de $\chi^{2}$ (Chicuadrado) para proporciones. Se utilizaron 32 registros correspondientes a las capturas realizadas en Magdalena y Punta Indio.

Termorregulación. En cada captura se registró la temperatura corporal del animal mediante la introducción vía rectal de un termómetro digital. Además se tomaron medidas de la temperatura y humedad atmosféricas utilizando un termo higrómetro. De esta manera, se lograron 47 registros de temperatura corporal, acompañados de los correspondientes registros de temperatura y humedad ambientales. Utilizando regresiones lineales simples se evaluó la relación entre la temperatura corporal (variable dependiente) contra las siguientes variables independientes: temperatura ambiente y logaritmo en base 10 del peso. Ambas resultaron significativas, por lo tanto las diferencias de temperatura corporal entre machos y hembras, y entre las distintas estaciones del año se evaluaron mediante análisis de covarianza (ANCOVA) utilizando, por separado, el logaritmo en base 10 del peso y la temperatura ambiente.

Reproducción. Para esta sección se tuvieron en cuenta las capturas pertenecientes a los campos de 
Magdalena y Punta Indio. Se utilizó la información proveniente de la identificación del sexo, la edad relativa (cría - juvenil - adulto) y la condición reproductiva. La edad relativa fue evaluada principalmente a partir del tamaño del individuo, y se tuvieron en cuenta además otros rasgos, como marcas, cicatrices y aspecto de la piel y coraza. La condición reproductiva fue definida evaluando ciertos rasgos corporales, tales como: en hembras, el estado de la vulva y la longitud de los pezones, esto último para reconocer las que pudieran estar amamantando; en machos, la notoriedad de los testículos en el abdomen, teniendo en cuenta que estos animales presentan testículos intraabdominales. Para calcular la proporción de sexos se utilizó la siguiente relación: $\mathrm{P}=$ cantidad de machos/cantidad de hembras, no se tuvieron en cuenta a las crías. La capturas analizadas fueron 29 y se evaluaron a partir de pruebas $\chi^{2}$ (Chi-cuadrado).

Morfometría. Para esta sección se obtuvieron datos de 39 adultos (25 hembras y 14 machos), 11 juveniles (tres hembras y ocho machos) y 29 crías (todas hembras, 14 obtenidas durante los tres años correspondientes a este trabajo y 15 de muestreos más recientes en los mismos campos). Para las medidas se siguió a Loughry \& McDonough (1996) con algunas modificaciones: largo de cabeza (LCa), longitud de la oreja (LO), longitud de los bordes anterior (ET, escudo torácico) y posterior (FET, fin escudo torácico) del escudo torácico, longitud de la última banda móvil (UBM), largo del cuerpo (LCu), largo de la cola (LC), perímetro de la base de la cola (BC), y peso.

Las relaciones alométricas de crecimiento entre las medidas morfométricas externas y el peso transformados en logaritmos de base 10, se evaluaron mediante análisis bivariado de regresión lineal utilizando Standarized Major Axis (WARTON et al., 2006). Los desvíos del valor esperado para una relación isométrica de 0,333 (debido a que se involucran medidas de crecimiento lineal y cúbica), de los coeficientes alométricos (pendientes), se evaluaron mediante pruebas de $F$ utilizando el paquete smart en R (WARTON \& WEBER, 2002; WARTON $\&$ Ormerod, 2007), con un nivel global a $=0,05$ y un nivel individual $\mathrm{a}=0,00625$, resultante de aplicar la corrección de Bonferroni (0,05 dividido el número de pruebas estadísticas realizadas sobre la misma muestra; HAIR et al., 1995). Las diferencias en los residuos de la regresión entre ambos sexos se evaluaron mediante la prueba no paramétrica de Wilcoxon. Esta misma prueba estadística fue utilizada para evaluar la existencia de dimorfismo sexual dentro de cada grupo de edad relativa para cada variable morfométrica, incluyendo el peso.

\section{RESULTADOS}

Se obtuvieron un total de 71 capturas, repartidas en diferentes épocas del año en los distintos campos muestreados (Tab. I).

Hábitos alimentarios. Las categorías en las que se agruparon los restos registrados en las heces fueron las siguientes: Coleoptera (coleópteros adultos y larvas),
Formicidae (hormigas adultas, larvas y huevos), Blattaria (cucarachas), arañas (Clase Arachnida), ciempiés, mil pies (Miriápodos) y Orthoptera (langostas y grillos). Los vertebrados registrados fueron anfibios, reptiles y pequeños mamíferos. El material vegetal registrado consistió en hojas, raíces, tallos, bulbos, frutos y semillas (para el análisis se tratan agrupados). Los resultados generales se presentan en la Figura 2, para una mejor interpretación se relativizó cada porcentaje en volumen $(\% \mathrm{~V})$ al peso total de la feca. Asimismo, no fueron incluidos en la Figura 2 los ítems que no superaban el $1 \% \mathrm{~V}$, estos fueron vertebrados (pequeños mamíferos, reptiles y anfibios), miriápodos y arañas. El promedio del material que no se pudo determinar fue bajo $(3,1 \% \mathrm{~V})$, con valores que oscilaron entre el 0,3 a $13,5 \% \mathrm{~V}$. Los ítems principales de la dieta de la mulita incluyen material vegetal, seguidos por hormigas y coleópteros. El resto de los ítems se observa en un bajo porcentaje. No se registró dependencia significativa entre los ítems y la estación del año $\left(\chi^{2}=8,2239\right.$, g.l.= $27, \mathrm{p}>0,05)$, o sea, no existen diferencias significativas en la composición de la dieta por estación.

Uso del espacio y del tiempo. Se obtuvieron 19 capturas en verano, 17 en primavera, cinco en otoño y cuatro en invierno, observando una diferencia significativa en la cantidad de capturas por estación $\left(\chi^{2}=\right.$ $16,42$, g.l. $=3, p<0,0009)$. Las capturas se registraron durante todo el día.

En el análisis estacional de los horarios de captura no observamos un uso diferente del tiempo entre las estaciones (Fig. 3). Durante las estaciones frías la mulita pampeana concentra su actividad al mediodía con promedio angular (a) \pm desvío angular promedio (s) para otoño de 12:36:17 $\pm 1: 27: 09(\mathrm{n}=5)$ e invierno 12:50:32 \pm 1:04:29 $(\mathrm{n}=3)$. Mientras que durante las estaciones cálidas, si bien el horario medio de actividad se da durante el medio día con a $\pm \mathrm{s}$ para primavera de 15:10:06 \pm $2: 36: 41(n=26)$ y verano de 12:36:19 $\pm 3: 33: 49(n=11)$, las mulitas pampeanas tienen dos picos de actividad, uno por la mañana $\mathrm{a} \pm \mathrm{s}=12: 09: 18 \pm 1: 18: 52(\mathrm{n}=20)$ y otro por la tarde, 17:49:01 $\pm 0: 57: 51(n=17)$. Aunque los datos no siguen una distribución uniforme $(Z$ de Raileight $=22,9218$ vs. $Z$ crítico $=2,979)$ las diferencias entre estaciones resultaron no significativas de acuerdo a la prueba de comparaciones múltiples de WatsonWilliams $(F=1,6337$, vs. valor crítico $F(0,05,(1), 3$, $41)=2,8327, p=0,196)$. La comparación de WatsonWilliams entre ambos picos de las estaciones cálidas resultó significativa $(\mathrm{F}=236,3434$, vs. valor crítico $\mathrm{F}$ $(0,05,(1), 1,35)=4,1213, p<0,0001)$. Sólo una mulita fue capturada durante la noche de un día muy caluroso de verano $\left(24^{\circ} \mathrm{C}, 91 \%\right.$ humedad, 3 de febrero de 2009 , Magdalena).

Con respecto al tipo de suelo frecuentado se observa un mayor uso de los suelos húmicos (45 capturas) versus los arenoso-arcillosos (13 capturas). Ninguna mulita fue capturada en terrenos bajos. Con respecto al tipo y características de la vegetación, el $82,7 \%$ de las capturas (48) fueron realizadas en pastizales, de los cuales la gran mayoría eran densos y altos $(75 \%$, altura 


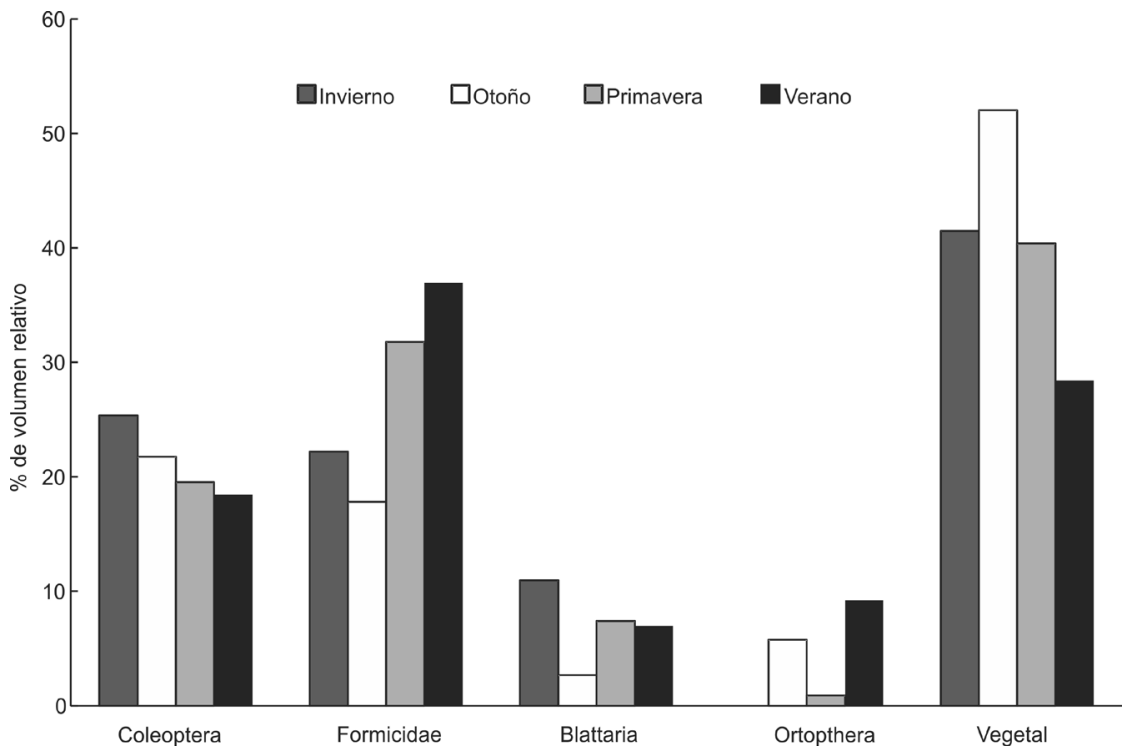

Fig. 2. Histograma con el porcentaje en volumen relativo al peso de la feca de Dasypus hybridus (Desmarest, 1804) por ítem y por estación.

de más de $20 \mathrm{~cm}$ y cobertura mayor al 50\%). El resto de las capturas se realizaron en montes naturales (cinco capturas) y en rastrojos de cultivos (cuatro capturas en soja y una en trigo), nunca se capturaron mulitas en pasturas implantadas.

Comportamiento. En la Figura 4 se muestran los resultados obtenidos del registro del "comportamiento inicial" observado en las diferentes estaciones del año, agrupadas en cálidas (primavera-verano) y frías (otoñoinvierno), destacándose que el 50\% de las mulitas capturadas en las estaciones frías estaban dentro de las cuevas.

En la comparación de la disponibilidad de ambientes versus el ambiente seleccionado para huir, montes $(14,4 \%)$ vs. pastizales $(85,5 \%)$, este último comprende tanto al pastizal natural $(76,4 \%)$ como pastura $(9,1 \%)$, se observaron diferencias significativas $\left(\chi^{2}=69,7\right.$, g.l. $\left.=1, p<0,0001\right)$, es decir, utilizan más los montes que lo esperado por el azar. La mayoría de las capturas fueron de individuos solitarios (44 de 55), las 11 capturas de más de un individuo adulto fueron parejas (macho y hembra, ver Reproducción).

Termorregulación. De los 47 registros de temperatura rectal, cinco correspondieron a invierno, seis a otoño, 21 a primavera y 15 a verano. La temperatura promedio fue de $35,96^{\circ} \mathrm{C}$ con una máxima de $38,4^{\circ} \mathrm{C}$ y una mínima de $33,1^{\circ} \mathrm{C}$. Se obtuvo una correlación positiva entre la temperatura rectal y la temperatura ambiental (Fig. 5, $\mathrm{T}^{\mathrm{o}}$ ambiental: $\mathrm{R}^{2}=0,33, \mathrm{~F}_{(1,34)}=16,6$, $\mathrm{p}<0,0002)$, sin embargo, la temperatura ambiental solo explica el 33\% de la variación de la temperatura rectal. Por el contrario, no se encontró correlación entre la temperatura rectal y el logaritmo decimal del peso $\left(R^{2}=0,079, F_{(1,34)}=2,91, p=0,097\right)$. El ANOVA muestra que hay diferencias significativas entre las temperaturas rectales tomadas en las distintas estaciones del año $\left(\mathrm{F}_{(3,32)}=4,8877, \mathrm{p}=0,00657\right)$, registrándose temperaturas rectales más altas en la estación más cálida (verano $=36,7^{\circ} \mathrm{C}$ ) que en la estación más fría (invierno $=34,4^{\circ} \mathrm{C}$; Fig. 6). La Tabla II muestra los resultados de los ANCOVA de la temperatura rectal para cada factor considerado utilizando la temperatura ambiental como covariable. Se detectaron diferencias para el comportamiento (Tab. II), registrándose temperaturas rectales más altas cuando el animal se encontraba activo, con una media considerando el efecto de la temperatura ambiente (MceTa) de $36,6^{\circ} \mathrm{C}$, sin embargo, cuando el animal se encontraba en reposo la temperatura rectal (MceTa) era de $35,5^{\circ} \mathrm{C}$ (Fig. 7). También para la edad se observaron diferencias (Tab. II), registrando menores temperaturas rectales en las crías $\left(\mathrm{MceTa}=34,7^{\circ} \mathrm{C}\right)$ que en juveniles $\left(\mathrm{MceTa}=36,35^{\circ} \mathrm{C}\right)$ y adultos $(\mathrm{MceTa}$

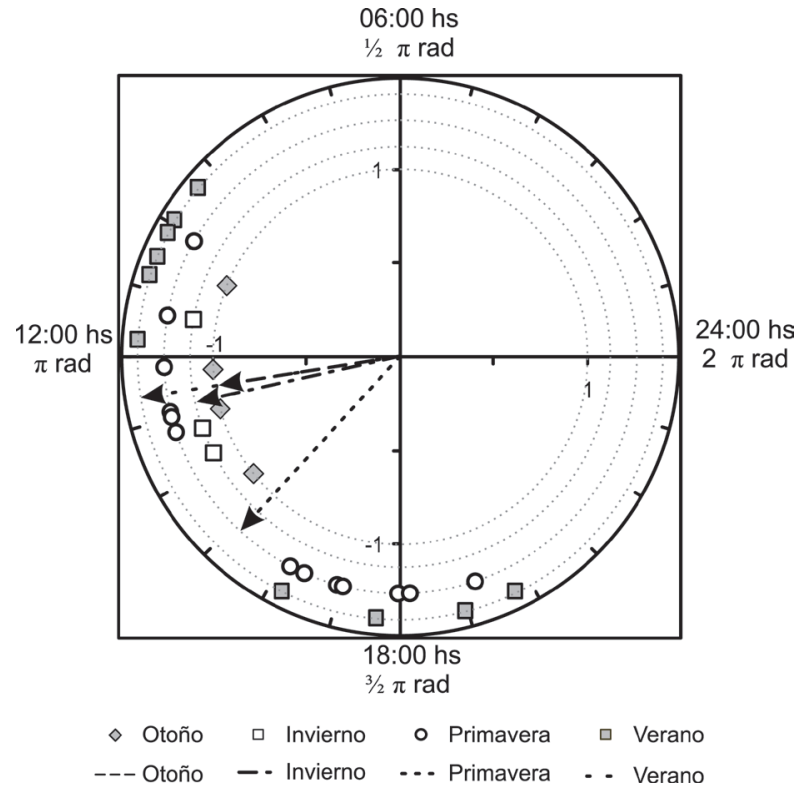

Fig. 3. Gráfico de dispersión del uso del tiempo de Dasypus hybridus (Desmarest, 1804) transformado a una distribución angular. Las líneas representan las medias de cada estación y las figuras los registros de los distintos horarios transformados en radianes. 


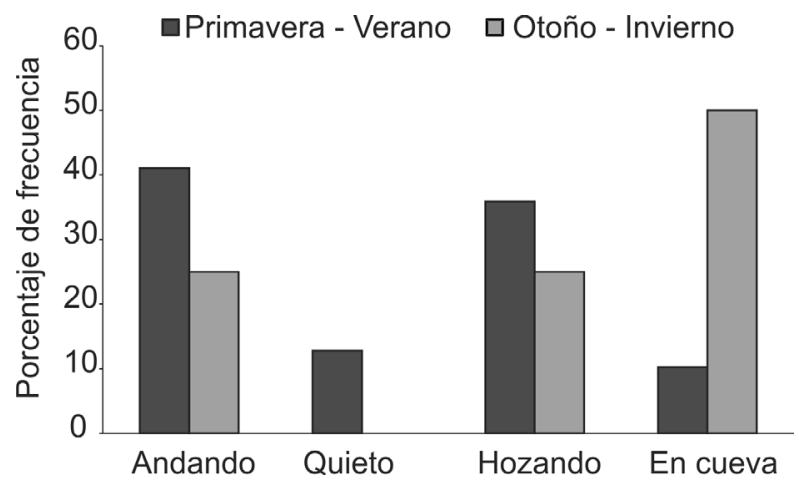

Fig. 4. Histograma con el porcentaje de frecuencias de pauta comportamental registrada para Dasypus hybridus (Desmarest, 1804) antes de la captura agrupado por estaciones cálidas (primavera y verano) y frías (otoño e invierno), en la Provincia de Buenos Aires, Argentina.

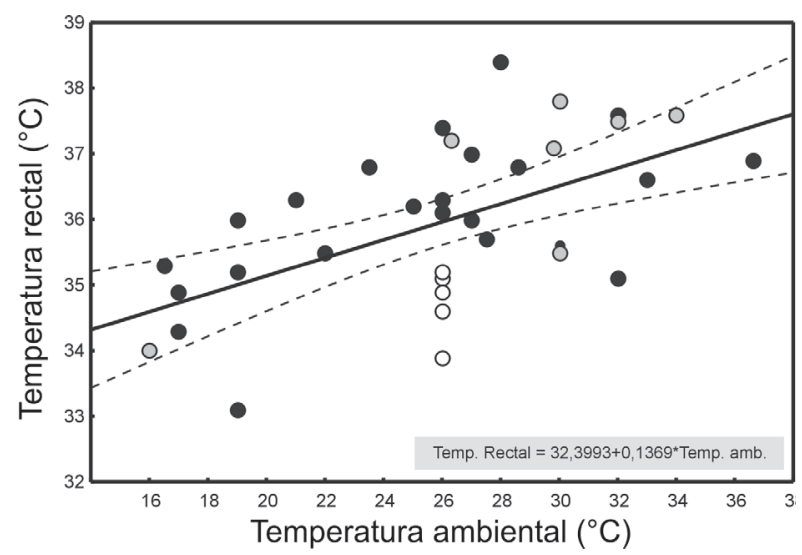

Fig. 5. Regresión de la temperatura rectal de Dasypus hybridus versus la temperatura ambiente (línea continua, recta de regresión; líneas de puntos, banda de 95\% confianza; círculos blancos, crías; círculos grises, juveniles; discos negros, adultos).

$=36,14^{\circ} \mathrm{C}$, Fig. 8). Por el contrario, no se observaron diferencias entre los sexos (Tab. II; Fig. 9).

Reproducción. La proporción de sexos fue igual a $0,81(\mathrm{P}=13 / 16)$, resultado similar a lo esperado $\left(\mathrm{P}=1, \chi^{2}=0,333\right.$, g.l. $\left.=1, \mathrm{p}<0,563703\right)$. Fueron capturadas cinco hembras que tenían evidencias de estar dando de mamar, todas en primavera. En cinco ocasiones se capturaron parejas (un macho y una hembra) interactuando entre sí (se desplazaban juntas, cruzándose y entrando en contacto directo durante varias ocasiones), dos en primavera (meses de octubre y principios de diciembre) y tres en verano (meses de diciembre y febrero). Se capturaron seis hembras con evidencias de preñez, todas en primavera (meses de octubre, noviembre y diciembre). En dos ocasiones se capturaron crías (ambas a finales de la primavera, 14 y 15 de diciembre), una camada de seis y otra de ocho individuos, en ambos casos las capturas se llevaron a cabo dentro de cuevas con abundante pasto.

Morfometría. En la Tabla III se presentan los promedios de las medidas tomadas ordenados por edad relativa y sexo, para este último sólo se consideran los adultos. Las camadas de crías fueron consideradas por separado en esta misma tabla. Todas las regresiones de las variables morfométricas externas resultaron significativas después de aplicar la corrección de Bonferroni ( $\mathrm{p}<0,00625$; Tab. IV y Fig. 10). De las ocho variables consideradas, la longitud de la oreja es la que posee el coeficiente de determinación más bajo $\left(\mathrm{R}^{2}=0,93\right.$; Tab. IV) y mayor dispersión alrededor de la línea de regresión (Fig. 10). La longitud del cuerpo, el borde posterior del escudo escapular y la última banda móvil son las variables que mejor correlacionan y menor dispersión alrededor de la recta de regresión poseen (Tab. IV). En el análisis alométrico, las pendientes de todas las variables difieren significativamente del valor de un tercio $(0,33)$ esperado para una ley de crecimiento isométrico. Por lo tanto, las variables, largo del cuerpo, base de la cola, borde posterior del escudo escapular y última banda móvil siguen un crecimiento alométrico positivo, es decir con pendiente significativamente mayor a un tercio $(0,33)$, a diferencia del resto de las variables que siguen una tendencia alométrica negativa (Tab. IV). No se observó dimorfismo sexual dentro de los juveniles y adultos, todas las pruebas de Wilcoxon resultaron no significativas (Tab. V).

\section{DISCUSIÓN}

Hábitos alimentarios. Lo primero a destacar en lo que refiere a los hábitos alimentarios es la alta cantidad de material vegetal encontrada en la dieta; muy posiblemente parte de este material sea accidental y no realice un aporte significativo a la dieta, pero con la metodología aplicada es difícil poder definir esta cuestión. Actualmente se están colectando individuos atropellados de esta especie y se están realizando nuevos análisis de dieta con estómagos; estas muestras arrojan las tendencias aquí esbozadas y la reportada en la bibliografía (ABBA \& CASSINI, 2010), o sea, es una especie omnívora con una marcada tendencia hacia la mirmecofagia. La inexistencia de una estacionalidad en la dieta se puede deber al bajo número de muestras analizadas, sin embargo, aunque sin una potencia estadística, podemos destacar un alto consumo de hormigas (tanto adultos como larvas y huevos) durante las estaciones cálidas.

Para esta especie, el único dato lo aporta NeGRETE et al. (datos inéditos) en un trabajo preliminar sobre anfibios y reptiles en la dieta de tres especies de dasipódidos, aquí indican que $D$. hybridus consume Amphisbaena sp. (Squamata, Amphisbaenidae), dato confirmado en nuestro trabajo. Además se registraron otros vertebrados como pequeños mamíferos y anfibios, sin embargo la proporción en la dieta fue muy baja (ver Resultados) por lo cual no se tuvieron en cuenta en el análisis final.

Las otras especies de Dasypus sobre las que se poseen datos de los hábitos alimentarios son $D$. kappleri Krauss, 1862, D. sabanicola Mondolfi, 1968, D. septemcinctus Linnaeus, $1758 \mathrm{y}$, mayormente, D. novemcinctus (SILVA, datos inéditos; McDonough \& Loughry, 2008). Las dos primeras especies 
Tab. II. Análisis de covarianza de la temperatura rectal de Dasypus hybridus para cada variable categórica utilizando la temperatura ambiente como covariable.

\begin{tabular}{lcccc}
\hline \multirow{2}{*}{ Variable categórica } & Categorías (n) & \multicolumn{3}{c}{ ANCOVA } \\
\cline { 3 - 5 } Sexo & Hembra (21), Macho (15) & F & g.1. & p \\
Edad & Cría (6), Juvenil (7), Adulto (23) & 7,0964 & $1 ; 33$ & 0,9223 \\
Comportamiento & Activo (17), Reposo (19) & 16,742 & $1 ; 33$ & 0,0028 \\
\hline
\end{tabular}

Tab. III. Promedios de las medidas de Dasypus hybridus (en milímetros y gramos) ordenados por edad relativa, sexo (sólo se considera los adultos) y camadas (grupos de crías) (LCa, largo cabeza; LO, longitud oreja; ET, escudo torácico; FET, fin escudo torácico; UBM, última banda móvil; $\mathrm{LCu}$, largo cuerpo; BC, base cola; LC, largo cola).

\begin{tabular}{lccccccccc}
\hline Edad - Sexo - n & LCa & LO & ET & FET & UBM & LCu & BC & LC & Peso \\
\hline Adultos (39) & 83,03 & 26,17 & 162,21 & 269,08 & 264,05 & 255,57 & 104,95 & 163,10 & 2006,3 \\
Juveniles (11) & 80,20 & 25,50 & 144,80 & 231,70 & 224,10 & 231 & 91,40 & 150,20 & 1134 \\
& & & & & & & & & \\
Machos (14) & 83 & 26,62 & 164,21 & 270,21 & 265,93 & 248,67 & 106,64 & 164,57 & 2002,8 \\
Hembras (25) & 83,04 & 25,91 & 161,08 & 268,44 & 263 & 259,17 & 104 & 162,28 & 2008,3 \\
& & & & & & & & & \\
Camada I (6) & 61,83 & 21,33 & 113,33 & 161,5 & 151,16 & 159 & 58 & 104,83 & 396,6 \\
Camada II (8) & 46,62 & 16 & 64,87 & 85,37 & 77,75 & 89,62 & 31,62 & 63,37 & 89,37 \\
\hline
\end{tabular}

prácticamente basan su dieta en insectos coleópteros, unos pocos vertebrados y no consumen plantas (ver McDonough \& Loughry, 2008). La dieta de $D$. septemcinctus se compone principalmente de hormigas y termitas (aproximadamente 60\%), otros artrópodos y una gran proporción de semillas y fragmentos vegetales (poco menos del 35\%). Dasypus novemcinctus presenta una gran variación en sus hábitos alimentarios, tanto geográfica como estacionalmente; en este amplio rango encontramos consumos del $70 \%$ de plantas hasta el $92 \%$
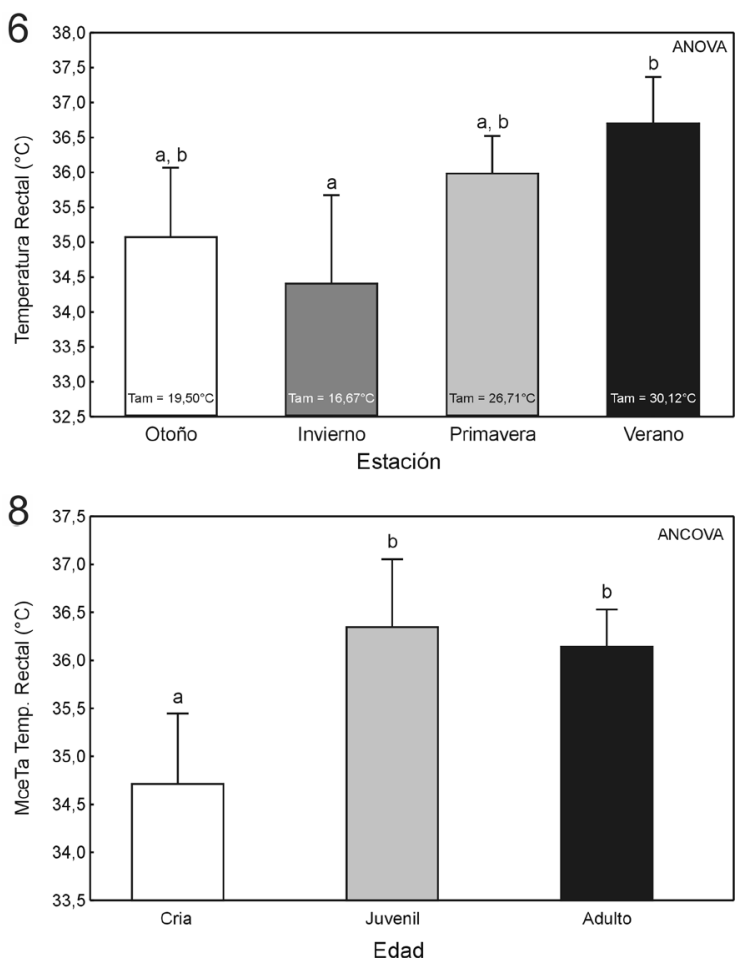

de himenópteros, pero los vertebrados nunca superan el 25\% (ver McDonough \& Loughry, 2008). Los datos obtenidos para $D$. hybridus se asemejan, en sus rasgos generales, a los observados en $D$. septemcinctus, destacándose una marcada tendencia a la mirmecofagia, o sea al consumo de hormigas/termitas. Por lo tanto estas dos especies se diferencian de las restantes del género ya que tienden a ser insectívoros especialistas.

REDFORD (1985) en un trabajo de revisión de los tipos de dieta de los armadillos, incluye al género
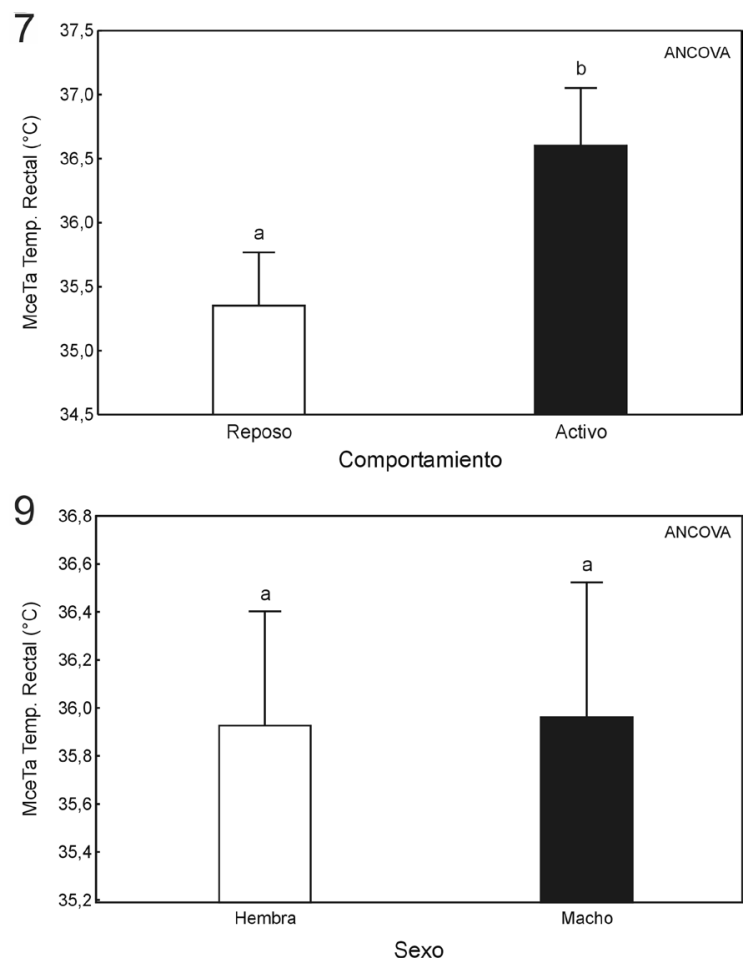

Figs. 6-9, Dasypus hybridus (Desmarest, 1804), análisis de temperatura rectal: 6, ANOVA de la temperatura rectal versus las estaciones del año; 7 a 9, ANCOVA de la temperatura rectal versus comportamiento, edad y sexo utilizando la temperatura ambiente como covariable (Tam, temperatura ambiente media por estación; MceTa, media considerando el efecto de la temperatura ambiente). 
Tabla IV. Resumen de estadísticos descriptivos para las regresiones simples obtenidas al utilizar los logaritmos decimales del peso de Dasypus hybridus (en gramos) como variable independiente y las variables morfológicas externas como variable dependiente (Variables: LCa, largo cabeza; LO, longitud oreja; LCu, largo cuerpo; LC, largo cola; BC, base cola; ET, escudo torácico; FET, fin escudo torácico; UBM, última banda móvil; $\mathrm{n}$, número de observaciones; R2 , coeficiente de determinación; $\mathrm{F}$, test regresión significativa; $p$, probabilidad; $\mathrm{O}$, ordenada al origen; 0.95 , $\mathrm{X}$ 95\% confianza; $t$, test de $\mathrm{t}$ ordenada diferente de cero; $\mathrm{P}$, pendiente; $\mathrm{F}$, test de $\mathrm{F}$ pendiente significativa diferente de 1 isometría; piso, valor $\mathrm{p}$ de la prueba $\mathrm{F}$ de isometría; Tend, tendencia).

\begin{tabular}{|c|c|c|c|c|c|c|c|c|c|c|c|c|}
\hline Var. & $\mathrm{n}$ & $\mathrm{R}^{2}$ & $\mathrm{~F}_{(1, \mathrm{n}-2)}$ & $p$ & $\mathrm{O}$ & 0.95 & $t_{(\mathrm{n}-2)}$ & $\mathrm{P}$ & $\mathrm{F}_{(1, \mathrm{n}-2)}$ & g.1. & piso & Tend \\
\hline $\mathrm{LCa}$ & 69 & 0,966 & 1898,66 & $<10^{-51}$ & 1,223 & 0,0273 & 89,397 & 0,21 & 441,621 & 67 & $<10^{-25}$ & - \\
\hline LO & 67 & 0,930 & 869,15 & $<10^{-39}$ & 0,851 & 0,0324 & 52,477 & 0,17 & 464,343 & 65 & $<10^{-25}$ & - \\
\hline $\mathrm{LCu}$ & 67 & 0,981 & 3401,29 & $<10^{-58}$ & 1,249 & 0,0341 & 73,118 & 0,35 & 9,540 & 65 & 0,003 & + \\
\hline $\mathrm{LC}$ & 75 & 0,954 & 1511,09 & $<10^{-50}$ & 1,206 & 0,0444 & 54,160 & 0,31 & 11,172 & 73 & 0,001 & - \\
\hline $\mathrm{BC}$ & 75 & 0,978 & 3305,12 & $<10^{-62}$ & 0,842 & 0,0355 & 47,248 & 0,36 & 17,637 & 73 & $<10^{-05}$ & + \\
\hline ET & 74 & 0,977 & 2995,11 & $<10^{-60}$ & 1,263 & 0,0302 & 83,293 & 0,29 & 62,246 & 72 & $<10^{-11}$ & - \\
\hline FET & 74 & 0,988 & 5975,42 & $<10^{-71}$ & 1,268 & 0,0263 & 96,097 & 0,35 & 20,601 & 72 & $<10^{-05}$ & + \\
\hline UBM & 74 & 0,990 & 6830,82 & $<10^{-73}$ & 1,197 & 0,0259 & 91,993 & 0,37 & 84,967 & 72 & $<10^{-14}$ & + \\
\hline
\end{tabular}

Dasypus en el grupo de insectívoros generalistas de hábitos terrestres, y los define como animales que se alimentan básicamente de insectos, pero que también comen vertebrados, huevos y material vegetal, sin embargo plantea que según su morfología y biología tendrían una tendencia a la mirmecofagia (o sea, a ser insectívoros especialistas). De esta manera REDFORD (1985) ubica a este género ocupando todo el rango de insectivoría (de generalista a especialista, ver Tabla I en REDFORD, 1985), sin especificar las posiciones ocupadas en ese rango por cada especie. Los datos recabados en nuestro trabajo permiten clarificar el panorama planteado por REDFORD (1985), destacándose la tendencia hacia la mirmecofagia de las mulitas de menor tamaño (D. septemcinctus y D. hybridus), con todo lo que esto conlleva (REISs, 2000). En contraposición, las especies de mayor tamaño (D. kappleri, D. sabanicola y $D$. novemcinctus) se corresponden con una dieta de insectívoro generalista.
Uso del espacio y del tiempo. Lo observado con respecto al uso del tiempo nos hace definir a esta especie como netamente diurna, confirmando lo observado por CASANAVE et al. (2003). Es de destacar que se obtuvieron pocas capturas de mulitas activas durante las estaciones frías, lo que hace suponer una baja sustancial en la actividad de estos armadillos durante los períodos fríos. Si tenemos en cuenta además los datos de termorregulación y comportamiento (ver más adelante), esta especie podría estar utilizando la estrategia de hibernar o entrar en torpor, como ya fue registrada en otra especie de armadillo, Zaedyus pichiy Desmarest, 1804 (Superina \& Boily, 2007).

Este estudio confirma la asociación de D. hybridus con los pastizales naturales ya presentada por varios autores (GonZÁlez et al., 2001; ABBA et al., 2007; ABBA \& CAssini, 2008), pero también la vincula con áreas de cultivo intensivo como son los campos de soja y trigo muy extendidos en el área de ocurrencia de esta especie.

Tabla V. Estadísticos descriptivos de 11 variables morfométricas externas de Dasypus hybridus (mm) y el peso (gramos) discriminados por edad y por sexo. Las pruebas no paramétricas de Wilcoxon (Wil.) resultaron no significativas (LCa, largo cabeza; LO, longitud oreja; LCu, largo cuerpo; LC, largo cola; BC, base cola; ET, escudo torácico; FET, fin escudo torácico; UBM, última banda móvil).

\begin{tabular}{|c|c|c|c|c|c|c|c|c|c|}
\hline \multirow{2}{*}{ Edad } & \multirow{2}{*}{ Variable } & \multicolumn{3}{|c|}{ Femenino } & \multicolumn{3}{|c|}{ Masculino } & \multirow{2}{*}{ Wil. } & \multirow{2}{*}{$p$} \\
\hline & & Media & $\mathrm{sd}$ & $\mathrm{n}$ & Media & $\mathrm{sd}$ & $\mathrm{n}$ & & \\
\hline \multirow{9}{*}{ Juvenil } & Peso & 1245,00 & 7,071 & 2 & 1185,71 & 385,697 & 7 & 9,5 & 0,557 \\
\hline & $\mathrm{LCa}$ & 78,00 & 0,000 & 2 & 82,50 & 0,707 & 2 & 0 & 0,221 \\
\hline & LO & 26,00 & 1,414 & 2 & 24,50 & 0,707 & 2 & 3,5 & 0,414 \\
\hline & $\mathrm{LCu}$ & 222,50 & 3,536 & 2 & 260,00 & 14,142 & 2 & 0 & 0,333 \\
\hline & $\mathrm{LC}$ & 165,50 & 16,263 & 2 & 143,33 & 29,924 & 6 & 8 & 0,615 \\
\hline & $\mathrm{BC}$ & 98,50 & 0,707 & 2 & 91,50 & 8,526 & 6 & 10 & 0,286 \\
\hline & ET & 132,50 & 2,121 & 2 & 149,50 & 8,313 & 6 & 0 & 0,065 \\
\hline & FET & 231,00 & 4,243 & 2 & 235,17 & 17,815 & 6 & 6 & 1,000 \\
\hline & UBM & 217,50 & 2,121 & 2 & 229,17 & 18,627 & 6 & 3,5 & 0,502 \\
\hline \multirow{9}{*}{ Adulto } & Peso & 2008,33 & 382,175 & 24 & 2002,86 & 171,528 & 14 & 190,5 & 0,505 \\
\hline & $\mathrm{LCa}$ & 82,77 & 3,116 & 22 & 83,00 & 3,530 & 14 & 146,5 & 0,819 \\
\hline & LO & 25,95 & 1,284 & 21 & 26,62 & 1,609 & 13 & 105,5 & 0,264 \\
\hline & $\mathrm{LCu}$ & 258,95 & 23,014 & 22 & 248,67 & 11,919 & 12 & 181,5 & 0,077 \\
\hline & $\mathrm{LC}$ & 160,75 & 15,394 & 24 & 164,57 & 9,851 & 14 & 147 & 0,534 \\
\hline & $\mathrm{BC}$ & 103,25 & 7,914 & 24 & 106,64 & 6,320 & 14 & 130,5 & 0,261 \\
\hline & ET & 161,83 & 9,126 & 24 & 164,21 & 9,167 & 14 & 145 & 0,495 \\
\hline & FET & 267,29 & 16,779 & 24 & 270,21 & 10,024 & 14 & 163,5 & 0,904 \\
\hline & UBM & 262,96 & 17,087 & 24 & 265,93 & 9,286 & 14 & 165,5 & 0,952 \\
\hline
\end{tabular}



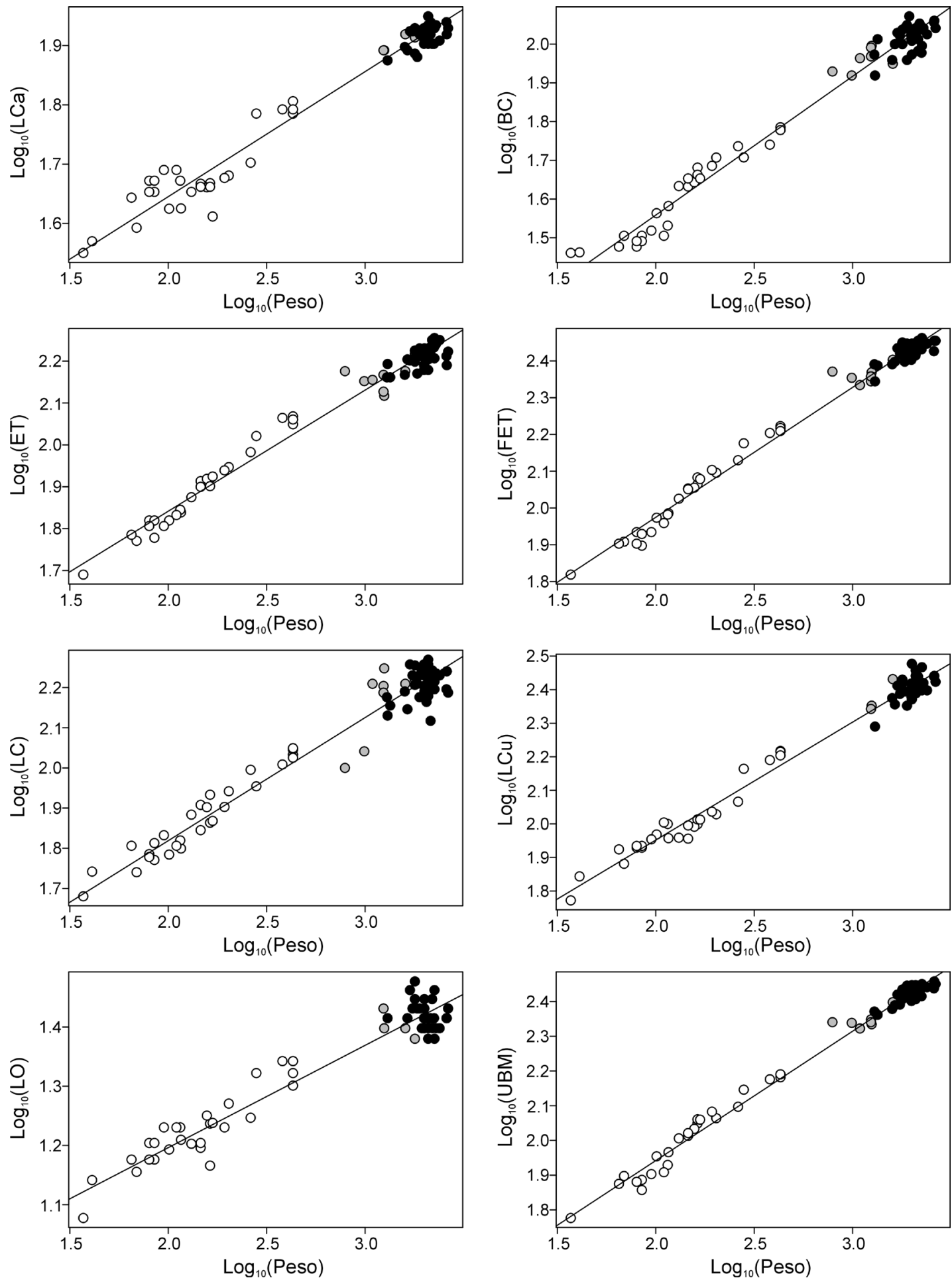

Fig. 10. Relaciones alométricas de las medidas externas de Dasypus hybridus (Desmarest, 1804) con respecto al peso corporal (discos negros, adulto; discos grises, juvenil; círculos blancos, cría; LCa, largo cabeza; LO, longitud oreja; ET, escudo torácico; FET, fin escudo torácico; UBM, última banda móvil; LCu, largo cuerpo; BC, base cola; LC, largo cola).

Comportamiento. Esta especie de mulita no escapa a la regla general de los armadillos (McDonough \& LOUGHRY, 2008): es solitaria y asocial, sólo se la observa interactuando en épocas de reproducción y cría. Al igual que lo observado en las otras dos especies que coexisten en estos ambientes (Chaetophractus vellerosus y $C$. villosus Desmarest, 1804, ver ABBA \& CASSINI, 2008 y
Авва et al., 2011), la mulita pampeana aprovecha las escasas áreas arboladas presentes en su hábitat (montes) y las utiliza como refugio. La disminución drástica de la actividad durante las estaciones frías sugiere la utilización de la hibernación y/o torpor como estrategia termorregulatoria (ver más arriba).

Termorregulación. Lo observado en esta sección 
básicamente muestra una relación directa de la temperatura rectal con la temperatura ambiental y con el comportamiento. Los valores medios y extremos registrados en $D$. hybridus no escapan a lo observado en otras especies de armadillos (ver CubA-CAPARO, 1976; McNAB, 1980, 1985).

Esta especie presenta ciertas características que podrían relacionarse a la existencia de períodos de torpor, sin embargo, las temperaturas registradas no son tan bajas como las esperadas para esta estrategia (Geiser, 1998; Superina \& Boily, 2007). Por otro lado, existe una relación directa entre la temperatura rectal y la temperatura atmosférica y entre la primera y el comportamiento registrado, por lo tanto, creemos que para confirmar la existencia de torpor y/o hibernación en la mulita pampeana es necesaria la realización de estudios posteriores.

Reproducción. La bibliografía publicada sobre este tema en D. hybridus es básicamente de datos provenientes de cautiverio o registros anecdóticos. En ella se plantea que las cópulas comienzan en marzo (finales del verano, principios de otoño) y las crías nacen desde octubre hasta diciembre, con un período de diapausa de los embriones de tres meses y un período de gestación de 120 días, a partir de la implantación del embrión (FERRARI et al., 1997).

A partir de los datos obtenidos en esta sección podemos plantear que el período reproductivo se concentra en primavera, donde se registraron la mayor cantidad de hembras preñadas y todas las crías. A diferencia de lo citado por FERRARI et al. (1997), donde los nacimientos bajo condiciones de laboratorio son registrados de octubre a noviembre, se han encontrado hembras preñadas hasta diciembre, por lo que el período de nacimientos podría extenderse hasta finales de diciembre o principios de enero.

En Dasypus novemcinctus las parejas se registran prácticamente todo el año, con una mayor frecuencia durante los meses de verano (McDonough, 2000; LAYNE, 2003). Este mismo patrón se esboza en nuestros datos, lo que permite asemejar la estrategia reproductiva de la mulita de nueve bandas con la de la mulita pampeana.

Morfometría. Los resultados de esta sección muestran que no se observó dimorfismo sexual en las medidas corporales externas a lo largo de la ontogenia, esto coincide con los reportes previos de la ausencia de dimorfismo sexual aparente en la morfología externa de los armadillos (McDonough \& Loughry, 2008). Todas las medidas externas consideradas poseen una alta correlación con la masa corporal $\left(\mathrm{R}^{2}>0,93\right)$. Ninguna de las variables consideradas sigue un crecimiento isométrico a lo largo de la ontogenia. En particular, el fin escudo torácico (FET), la última banda móvil (UBM) y el largo cuerpo ( $\mathrm{LCu}$ ) son las variables que mejor se correlacionan con la masa corporal $\left(\mathrm{R}^{2}>0,98\right)$ y siguen una relación alométrica positiva con la misma. En cambio, el largo de la cabeza (LCa), la longitud de la oreja (LO), el escudo torácico (ET) y el largo de la cola (LC) siguen una relación alométrica negativa, de modo que las crías poseen dichos atributos proporcionalmente más grandes que los adultos. Visualmente es más notable en el largo de la oreja, siendo las crías más orejudas que los adultos (morfométricamente su pendiente es la menor de todas, con un valor de 0,17; Tab. IV). La relación entre estas variables muestra un patrón de crecimiento diferencial del cuerpo con respecto a la cabeza y la cola a lo largo de la ontogenia, siendo la primera proporcionalmente más grande en el adulto.

Aportes a la Conservación. Desde el punto de vista de la conservación, esta especie está categorizada, tanto a escala global (ABBA \& SuPERINA, 2010) como local (SuPERINA et al., en prensa), como Casi Amenazada (Near Threatened). Ya a principios del siglo XVIII Azara (AzArA, 1998) comenta que es un "manjar delicado" y que se la caza fácilmente. FernANDEZ (1915), como corolario de su obra sobre la embriología de esta especie, hace una observación sobre su intenso uso y dice: "es notorio que miles y miles de estos animales llegan a los mercados de las grandes ciudades y destruyéndose indiferentemente los machos y las hembras preñadas la especie bien pronto deberá extinguirse". Asimismo, comenta que en los últimos años se observa una disminución considerable. A fines del siglo XX, RoIG (1991) postula extinciones locales en el sudoeste de su distribución. Finalmente, AвBA et al. (2007), a partir de evidencias indirectas, destacan que esta especie depende de los pastizales naturales y evita las pasturas cultivadas, y se ve afectada tanto por el impacto antrópico (es más abundante en sitios más alejados de los centros urbanos), como por la cantidad de perros que se encuentran en los campos.

Desde el punto de vista de la conservación es importante destacar que en el presente trabajo se ha registrado a la mulita pampeana en ambientes altamente modificados por labores agrícolas, hecho hasta ahora no reconocido (ver ABBA \& CASSINI, 2008). Asimismo, en la época reproductiva más del $70 \%$ de las hembras adultas capturadas estaban preñadas, dato muy alentador para la supervivencia a mediano plazo de la especie.

En el presente trabajo se han realizado aportes sobre diferentes aspectos de la biología de Dasypus hybridus. Esto es relevante en cuanto que permite contrastar los hábitos de vida de esta especie con otras afines mejor conocidas, como D. novemcinctus. Además, el conocimiento exhaustivo de sus hábitos alimentarios y de comportamiento y de los ciclos circadianos y reproductivos, aportan importante información a la hora de plantear estrategias de conservación asociadas a esta especie.

Agradecimientos. A S. F. Vizcaíno y M. H. Cassini. A P. A. Gado, M. C. Ezquiaga y L. G. Pagano por su invaluable ayuda en el campo y laboratorio. A G. T. Navone y M. Lareschi, S. Merani, H. López y E. Etcheverry por su constante apoyo. A C. A. Galliari por su ayuda en la corrección y perfeccionamiento del manuscrito. Al Ministerio de Asuntos Agrarios por los permisos otorgados. A los dueños y trabajadores de los campos, muy especialmente a la familias Rudzic, Landa, Soks, Muñiz Barreto, Mongiardino y Shaw. A la Facultad de Ciencias Naturales y Museo (UNLP), CONICET, CIC, Idea Wild, Conservation International, Agencia Nacional de Promoción Científica y Tecnológica (BID PICT2010-1412) por el apoyo económico. Finalmente, agradecemos al editor y revisores por sus comentarios que ayudaron a enriquecer notablemente el manuscrito. 


\section{REFERENCIAS BIBLIOGRÁFICAS}

AbBa, A. M. \& CAssini, M. H. 2008. Ecology and conservation of three species of armadillos in the Pampas region, Argentina. In: Vizcaíno, S. F. \& Loughry, W. J. eds. The Biology of the Xenarthra. Gainesville, University of Florida Press. p. 300-305.

2010. Ecological differences between two sympatric species of armadillos (Xenarthra, Mammalia) in a temperate region of Argentina. Acta Theriologica 55(1):35-44.

AbBa, A. M. \& SuperinA, M. 2010. The 2009/2010 Armadillo Red List Assessment. Edentata 11(2):135-184.

AbBa, A. M.; Cassini, M. H. \& Vizcaíno, S. F. 2007. Effects of land use on the distribution of threes species of armadillos (Mammalia, Dasypodidae) in the pampas, Argentina. Journal of Mammalogy 88(2):502-507.

Abba, A. M.; Cassini, G. H.; Cassini, M. H. \& Vizcaíno, S. F. 2011. Historia natural del piche llorón Chaetophractus vellerosus (Mammalia: Xenarthra: Dasypodidae). Revista Chilena de Historia Natural 84:51-64.

AnACleto, T. C. S. 2007. Food habits of four armadillo species in the Cerrado Area, Mato Grosso, Brazil. Zoological Studies 46(4):529-537.

Anacleto, T. C. S. \& Marinho-Filho, J. 2001. Hábito alimentar do tatu-canastra (Xenarthra, Dasypodidae) em uma área de cerrado do Brasil central. Revista Brasileira de Zoologia 18(3):681-688.

Anacleto, T. C. S.; Diniz-Filho, J. A. F. \& Vital, M. V. C. 2006. Estimating potential geographic ranges of armadillos (Xenarthra, Dasypodidae) in Brazil under niche-based models. Mammalia 70:202-213.

Azara F. DE. 1998. Viajes por la América Meridional. Tomo I. Buenos Aires, Ediciones El Elefante Blanco. 238p.

BILENCA, D. \& MiÑARO, F. 2004. Identificación de áreas valiosas de pastizal en las pampas y campos de Argentina, Uruguay y sur de Brasil. Buenos Aires, Fundación Vida Silvestre Argentina. 323p.

Cabrera, A. \& Yepes, J. 1940. Mamíferos Sudamericanos. Buenos Aires, Cía. Argentina de Editores. 352p.

Casanave, E. B.; Manfredi, M. C. \& Luengos Vidal, E. M. 2003. Ecología comportamental de los armadillos en un pastizal serrano. EDIUNS, Actas de las II Jornadas del SO Bonaerense 3:1-8.

Cicchino, A. C. 2006. Diversidad de carábidos (Insecta, Coleoptera, Carabidae) en un talar joven de la Laguna Nahuel Rucá, partido de Mar Chiquita, provincia de Buenos Aires. In: MéridA, E. \& AтHOR, J. eds. Talares bonaerenses y su conservación. Buenos Aires, Fundación de Historia Natural Felix de Azara. p.137-145.

Cuba-CAPARó, A. 1976. Some hematologic and temperature determinations in the 7-banded armadillo (Dasypus hybridus). Laboratory Animal Science 26:450-455.

Fernandez, M. 1915. Die Entwicklung der Mulita. Revista del Museo de La Plata 21:1-519.

Ferrari, C. C.; Carmanchahi, P. D.; Bolkovic, M. L. \& Affanni, J. M. 1997. Breeding in captivity of the southern lesser long-nosed armadillo Dasypus hybridus (Edentata = Xenarthra: Dasypodidae). Zoocriaderos 2:1-5.

GeISER, F. 1998. Evolution of daily torpor and hibernation in birds and mammals: importance of body size. Clinical and Experimental Pharmacology and Physiology 25:736-740.

Gerschman de Pekelín, B. S. \& Schiapelli, R. D. 1963. Llave para la determinación de familias de arañas argentinas. Physis 24(67):43-72.

GonzÁlez, E. M.; Soutullo, A. \& Altuna, C. A. 2001. The burrow of Dasypus hybridus (Cingulata: Dasypodidae). Acta Theriologica 46:53-59.

GREEGOR, D. H. 1980. Diet of the little hairy armadillo, Chaetophractus vellerosus, of northwestern Argentina. Journal of Mammalogy 61:331-334.

Hair, J. F.; Anderson, E. E; Tatham, R. L. \& Black, W. C. 1995. Multivariate Data Analysis. 4 ed. New Jersey, Prentice Hall. 745p.

LAYNe, J. N. 2003. Armadillo, Dasypus novemcinctus. In: FeLDHAMER, G. A.; THOMpson, B. C. \& Chapman, J. A. eds. Wild mammals of North America: biology, management, and conservation. Baltimore, London, The Johns Hopkins University Press. p.75-97.

Loughry, W. J. \& McDonough, C. M. 1996. Are road kills valid indicators of armadillo population structure? The American Midland Naturalist 135:53-59.

McDonough, C. M. 2000. Social organization of nine-banded armadillos (Dasypus novemcinctus) in a riparian habitat. The American Midland Naturalist 144:139-151.

McDonough, C. M. \& Loughry, W. J. 2008. Behavioral ecology of armadillos. In: Vizcaíno, S. F. \& Loughry, W. J. eds. The Biology of the Xenarthra. Gainesville, University of Florida Press. p.281-293.

McNAB, B. K. 1980. Energetics and the limits to a temperate distribution in armadillos. Journal of Mammalogy 61:606-627.

1985. Energetics, population biology, and distribution of xenarthrans, living and extinct. In: Montgomery, G. G. ed. The evolution and ecology of armadillos, sloths and vermilinguas. Washington, London, Smithsonian Institution Press. p.219-232.

Mena, M. 2004. Aplicaciones de estadística circular a problemas de ciencias naturales. Buenos Aires, Akadia. 152p.

Morrone, J. J. \& Coscarón, S. 1998. Biodiversidad de artrópodos argentinos. Una perspectiva biotaxonómica. La Plata, Ediciones SUR. 599p.

Orrego Aravena, R. 1974. Insectos de la Pampa (Coleópteros). Santa Rosa, Gobierno de la Provincia de la Pampa. 166p.

Ottino, P. \& Giller, P. 2004. Distribution, density, diet and habitat use of the otter in relation to land use in the Araglin valley, Southern Ireland. Biology and Environment: Proceedings of the Royal Irish Academy 1(104b): 1-17.

R Development Core Team. 2010. R: A language and environment for statistical computing. Vienna, R Foundation for Statistica Computing. Disponible en: <http://www.R-project.org $>$. Acceso en: 09.09.2010.

REDFord, K. H. 1985. Food habits of armadillos (Xenarthra: Dasypodidae). In: Montgomery, G. G. ed. The evolution and ecology of armadillos, sloths and vermilinguas. Washington and London, Smithsonian Institution Press. p.429-437.

Redford, K. H. \& Eisenberg, J. F. 1992. Mammals of the Neotropics. The southern cone. Chicago, University of Chicago. v.2, 430p.

Reiss, K. Z. 2000. Feeding in Myrmecophagous mammals. In: Schwenk, K. ed. Feeding: Form, Function and Evolution in Tetrapod Vertebrates. New York, Academic Press. p.459-485.

Roig, V. G. 1991. Desertification and distribution of mammals in the southern cone of South America. In: Mares M. A. \& SChmidly, D. eds. Latin American Mammalogy. Norman, University of Oklahoma Press. p.239-279.

Soll Survey Staff. 1999. Soil Taxonomy. A basic system of soil classification for making and interpreting soil surveys. 2ed. Washington, U.S. Department of Agriculture. Agriculture Handbook n. 436. 871p.

SuPERINA, M. \& BoILy, P. 2007. Hibernation and daily torpor in an armadillo, the pichi (Zaedyus pichiy). Comparative Biochemistry and Physiology 148(4):893-898.

Superina, M.; AbBA, A. M. \& Vizcaíno, S. F. En prensa. Magnaorden Xenarthra. In: OjedA, R.; DíAz, G. \& Chillo, V. eds. Libro Rojo de los mamíferos de Argentina. Mendoza, SAREM.

Warton, D. I. \& Ormerod, J. 2007. Smatr: (Standardised) Major Axis Estimation and Testing Routines. R package version 2.1. Disponible en: <http://www.eco-stats.unsw.edu.au/Software. $\mathrm{html}>$. Acceso en: 09.09.2010.

Warton, D. I. \& Weber, N. C. 2002. Common slope tests for bivariate structural relationships. Biometrical Journal 44:161-174.

Warton, D. I.; Wright, I. J.; Falster, D. S. \& Westoby, M. 2006. A review of bivariate line-fitting methods for allometry. Biological Reviews 81:259-291.

Wetzel, R. M. 1985. Taxonomy and distribution of armadillos, Dasypodidae. In: Montgomery, G. G. ed. The evolution and ecology of armadillos, sloths and vermilinguas. Washington, London, Smithsonian Institution Press. p.23-46.

Wetzel, R. M.; Gardner, A. L. \& Redford, K. H. 2007. Order Cingulata. In: GARDNER, A. L. ed. Mammals of South America: Marsupials, Xenarthrans, Shrews and Bats. Chicago, University of Chicago Press. v.1, p.128-156.

ZAR, J. H. 1999. Biostatistical analysis. 4ed. New Jersey, Prentice Hall. $663 \mathrm{p}$.

Recebido em 14 de abril de 2010. Aceito em 20 de outubro de 2011. ISSN 0073-4721

Artigo disponível em: www.scielo.br/isz

Impresso e distribuído em 2012

Iheringia, Série Zoologia, Porto Alegre, 101(4):325-335, 30 de dezembro de 2011 